\title{
Caractérisations métallurgiques et mécaniques des couches nitrurées : relation microstructure-comportement
}

\author{
M.E. Djeghlal, N. Hamedi et L. Barrallier ${ }^{1}$
}

Laboratoire de Sciences et Génie des Matériaux, Département de Métallurgie, École Nationale Polytechnique, 10 avenue Pasteur, BP. 182, El-Harrach, Alger, Algeria

${ }^{1}$ Laboratoire Mécasurf, ENSAM, 2 cours des Arts et Métiers, 13617 Aix-en-Provence cedex 1 , France

\begin{abstract}
Résumé . Dans le but de comprendre et d'expliquer le comportement de couches nitrurées d'un acier faiblement allié sous sollicitations mécaniques de type fatigue de contact, une caractérisation métallurgique et mécanique de ces couches a été entreprise. Pour mener à bien cette étude, la microscopie électronique à balayage (MEB), la diffractométrie $X(D R X)$ a également été mise en œuvre pour la détermination des contraintes résiduelles ainsi que pour effectuer des analyses des profils des pics de diffraction. La caractérisation micro structurale des couches nitrurées a permis l'identification de toutes les populations de précipités présents dans le matériau de base ainsi que la couche nitrurée elle-même .Les fractions volumiques ainsi que les distributions de tailles des familles de précipités ont été déterminées en fonction de la profondeur. Ces paramètres permettent ensuite de calculer le durcissement structural induit dans le matériau par la précipitation des nitrures .
\end{abstract}

Par cette étude nous allons essayer de mettre en évidence l'état structural du 32CDV13 nitruré à une température et temps de traitement $\mathrm{TN}=520^{\circ} \mathrm{C}, \operatorname{tn}=96 \mathrm{~h}$, la température de trempe est de $900^{\circ} \mathrm{c}$ ,du revenu est $\mathrm{TR}=600^{\circ} \mathrm{C}$, le taux de dissociation et entre 15 et $18 \%$.

\section{INTRODUCTION}

La nitruration est un traitement thermochimique dans lequel on fait diffuser de l'azote de la surface vers le cceur de la pièce à une température $T_{N}$ (température de nitruration) comprise entre 350 et $580^{\circ} \mathrm{C}$. Ce traitement permet de conférer une résistance mécanique importante aux pièces traitées. Dans le cas des aciers, les pièces subissent initialement un traitement thermique de trempe et de revenu à haute température. En effet, lorsque des éléments de structure exigent des tolérances dimensionnelles de grande précision de l'ordre de $3 \%$, et nécessitent un traitement thermochimique de durcissement superficiel, la nitruration serait le traitement le plus adéquat car ce dernier s'effectue sans changement allotropique du fer, les déformations et le gonflement pouvant surgir restant très faibles.

Le choix de la nitruration gazeuse est justifié par le fait que ce type de traitement est le plus employé industriellement, notamment par Aubert et Duval en France * . Les gammes utilisées dans cette étude correspondent à des applications industrielles. [1]

Cette étude a nécessité l'évaluation de différentes méthodes d'analyse (MEB, Analyse d'images, Microdureté, DRX ). Néanmoins, nous nous contenterons de mettre le point sur l'analyse d'images d'où les graphes (figures 1 à 5 ).

Cette technique a été utilisée dans le but d'automatiser la détermination des fractions surfaciques des précipités intergramulaires à partir de coupes micrographiques en microscopie optique. [2]

\footnotetext{
* Société Française de traitement de surface (Paris).
} 


\section{Etude expérimentale}

Des coupes micrographiques ont été pratiquées parallèlement à la surface de la couche nitrurée dont l'épaisseur de nitruration a atteint les $300 \mu \mathrm{m}$ ce qui répond à la norme de nitruration conventionnelle, dans le but d'avoir une vision précise sur la surface de contact.

L'attaque au Nital (3\%, quelques secondes ), dans le cas de la nitruration est insuffisante pour l'obtention d'un bon contraste. Elle est suivie d'une attaque au bisulfite de sodium pendant une minute, qui va augmenter le contraste des précipités intra granulaires par rapport à la matrice environnante.

L'acquisition des images se fait en utilisant un microscope optique, muni d'une caméra CDD. Ces dernières sont stockées afin d'être traitées par le Logiciel d'analyse d'images OPTILABPRO de Graftek. Afin de traiter des images de façon homogène et d'automatiser cette tâche , une interface spécifique a été développée par l'intermédiaire du logiciel LABVIEW de National Instruments .

L'association du microscope optique à l'analyseur d'images nous permet de recueillir les informations suivantes [3] :

$>$ Mesure de taille de grains

$>$ Comptage d'inclusions, paramètres de formes

$>$ Mesures granulomètriques de particules

$>$ Tailles des grains et Proportions de phases .

\section{Résultats \& Interprétation}

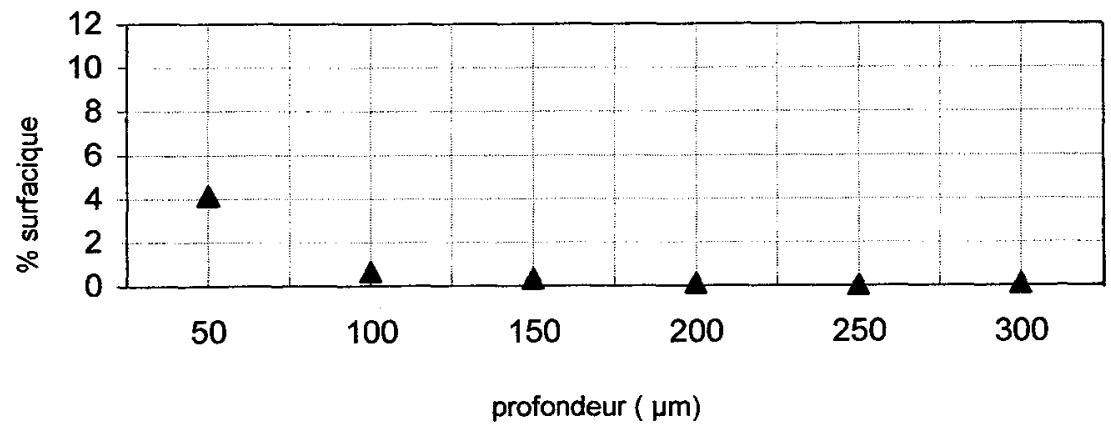

Figure 1: \%surfacique en fonction de la profondeur du 32CrMoV13 


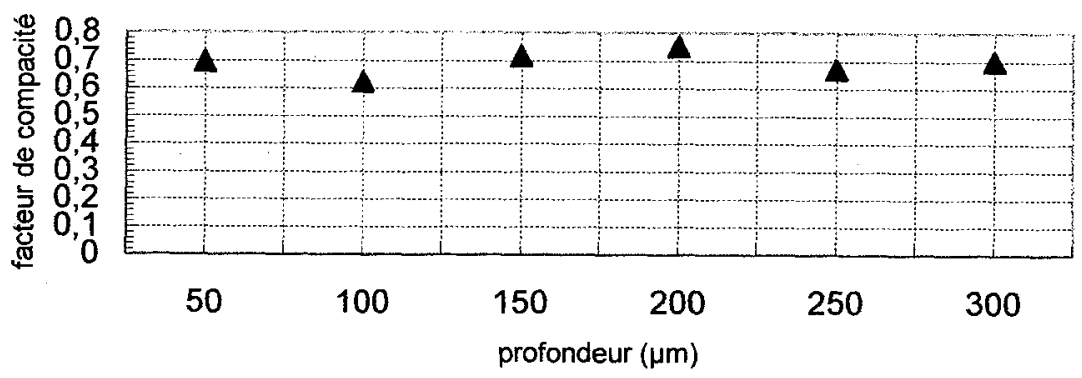

Figure 2 : Facteur de compacité en fonction de la profondeur dans le 32CrMoV13

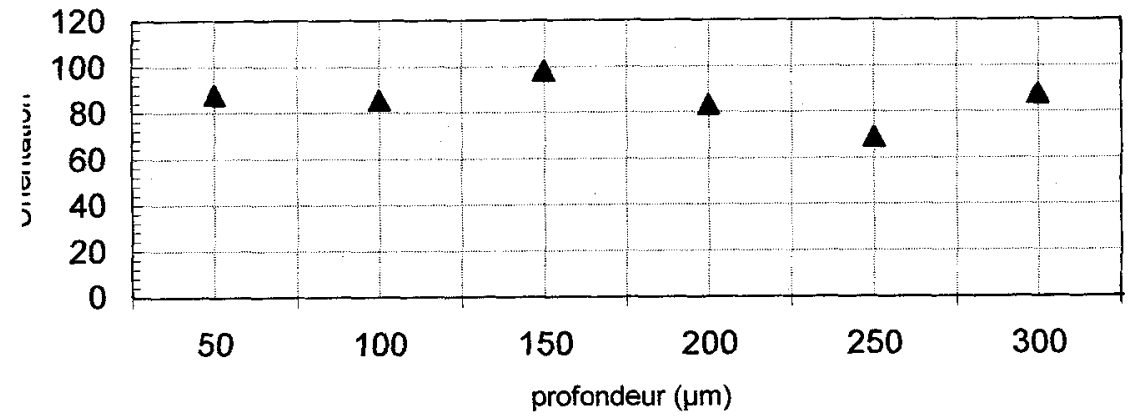

Figure 3 : Orientation en fonction de la profondeur du $32 \mathrm{Cr} \mathrm{MoV13}$

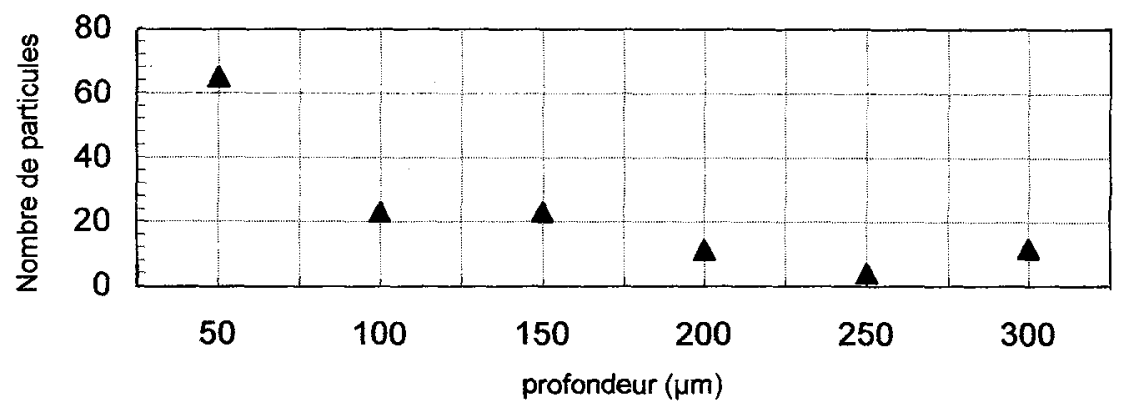

Figure 4 : Nombre de particules en fonction de la profondeur du $32 \mathrm{Cr}$ MoV13 


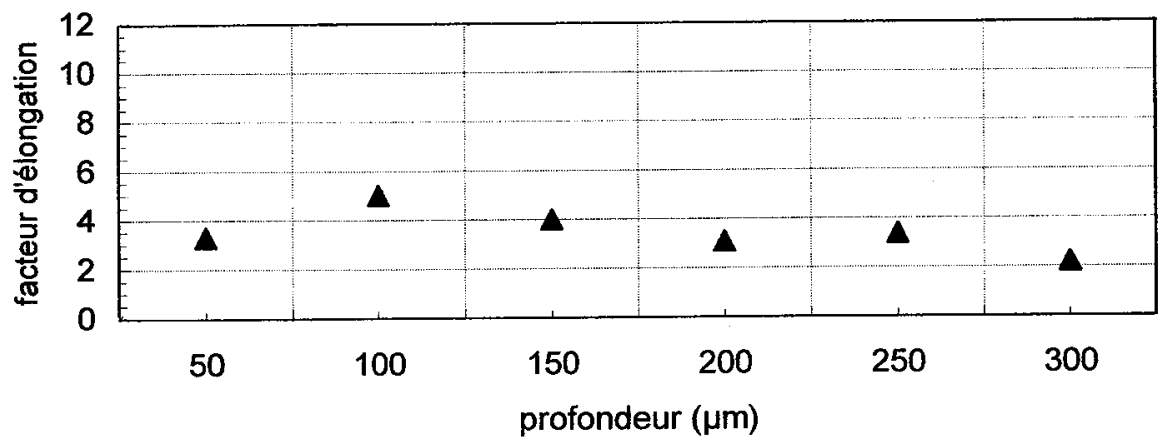

Figure 5 : Facteur d'élongation en fonction de la profondeur du 32CrMoV13

Cette analyse d'image a été entreprise pour essayer de quantifier la répartition des nitrures aux joints de grains dans la matrice .Dans l'apparition du phénomène de Pitting, la présence de $\varepsilon, \gamma^{\prime}$ nitrures de longueurs variantes, peut avoir une influence sur le comportement du matériau pendant le fonctionnement .La source de dégradation principale dans le cas des pignons étant la fatigue de contact, les couches superficielles jouent un rôle important et dans cette étude la première couche de 150 microns a été particulièrement étudiée.

En examinant nos résultats nous avons constaté que le \% surfacique représentant la surface de la particule /la surface scannée [3] est dégressif en fonction de la profondeur de la couche nitrurée (Figure 1), ce qui s'explique par le fait que les nitrures se forment de moins en moins que l'on s'éloigne de la surface de l'acier traité.

Le facteur de compacité Fc qui représente le rapport entre la surface de l'objet et la surface du plus petit rectangle contenant l'objet $\left(\mathrm{Fc}=\mathrm{A} / \mathrm{h}^{*} \mathrm{l}\right)$, est aussi important puisque il contribue à la détermination de la forme des nitrures.

Les orientations préférentielles des nitrures à partir de la mesure de la densité de nitrures [4], s'explique par le fait que les orientations préférentielles ( Figure 3) ,sont en relation d'orientation de BAKER -NUTTING [5], relative à : (010) du fer alpha et (001) du fer alpha . Par ailleurs, la forme des précipités n'est pas déterminée (disques ou rectangulaires), la preuve au MET n'est pas aisée à effectuer du fait de leur faible épaisseur, on peut néanmoins parler de désaccord suivant les directions privilégiées de $2,4 \%$ et $45 \%$ suivant (010) et (001). [6]

Nous pouvons dire que la nitruration profonde $96 \mathrm{~h}$ présente une forte carence de nitrures sur les premiers 50 microns, des contraintes de compression moindres que dans le cas de la nitruration conventionnelle. A partir de ces observations, il est possible de proposer une explication pour décrire la forte sensibilité au Pitting de la nitruration $96 \mathrm{~h}$, les contraintes en surface sont plus faibles et une dureté plus faible que la nitruration conventionnelle .

Du point de vue durcissement structural dû au traitement de nitruration, on peut dire que la nature et la composition des carbures présents dans le matériau de base ainsi que celles des populations de précipités présents dans la couche nitrurée ont été déterminées par le MET et le calcul thermodynamique utilisant THERMOCALC . Les distributions de tailles ont été déterminées en fonction de la profondeur, celles des nitrures globulaires font apparaître qu'ils possèdent des dimensions augmentant en fonction de la profondeur. 
A partir de ces observations, l'utilisation de l'analyse d'image pourrait être très utile pour quantifier la répartition des nitrures ;elle permettra de corréler l'observation microscopique des nitrures et les profils de micro dureté .Une quantification précise des états de surface des pièces semble inévitable car il ne fait aucun doute que lors du contact, la rugosité influe grandement sur la tenue en service .Les calculs thermodynamiques indiquent que le durcissement est apporté par un mécanisme d'Orowan [7] pour les nitrures globulaires et par un mécanisme de cisaillement pour les nitrures semi-cohérents.

\section{Références}

[1] L.Barrallier, Genèse des contraintes résiduelles de nitruration -Modélisation et expérimentation - , Thèse de doctorat ENSAM, (1992), pp.53.

[2] J.N.Locquet, Caractérisation Métallurgiques et Mécaniques de couches Nitrurées Relation Microstructure-Comportement, Thèse de doctorat ENSAM, (1998), pp.73.

[3] ENSAM, Guide de référence d'analyse d'image, (1990-1995).

[4] K.Alouache \& N.Ait Ben Hamou, Caractérisation et étude microstructurale des couches nitrurées par voie gazeuse de la nuance d'acier 32CDV13, Projet de fin d'études, (1998), pp.59.

[5] Baker .R.G.,Nutting J., Precipitation process in steels, I.S.1 Special Report ,(1968), pp.64.

[6] V.A. Philips A.U Seybolt., A Transmission Electron Microscopic Study of some ironNitrited Binary iron alloys and steels, Trans. of the metallurgical society of AIME, (1968), pp. 2415-2442.

[7] L.M.Brown R.K .Ham., Stengthening methods in crystals, Applied Science publishers Barking, (1971), pp.10-129. 